Onkologie 1991;14(suppl 1):1

\title{
Impressum, Vol. 14, Supplement 1, 1991
}

Internationale Zeitschrift für Krebsf orschung und -behandlung

Herausgeber

S. Karger

Verlag für Medizin und Naturwissenschaften $\mathrm{GmbH}$,

Postfach 1724, D-8034 Germering (BRD)

Presserechtlich verantwortlich: Walter Kunz, Gesellschafter

Offizielles Organ

der Deutschen Gesellschaft für Hämatologie und Onkologie, der Österreichischen Gesellschaft für Hämatologie und Onkologie, der Österreichischen Krebsgesellschaft-Krebsliga

unter Fortfühung der «Österreichischen Zeitung für Onkologie»

Schriftleitung

W. Queißer, Mannheim

Tachschriftleitung

W. Berdel, Berlin

E. Dühmke, Göttingen

N. Jaeger, Bonn

M. Kaufmann, Heidelberg

P. Schlag, Heidelberg

Wissenschaftlicher Beirat

W. Berdel, Berlin V. Diehl, Köln C.H. Dittrich, Wien P. Drings, Heidelberg E. Dühmke,

Göttingen S. Eckhardt, Budapest H. Ehrhart, München H.H. Fiebig, Freiburg A. Gläser, Halle R.

Hartenstein, München K. Havemann, Marburg K.P. Hellriegel, Berlin R. Herrmann, Berlin H.W.

von Heyden, Einbeck D. Hoelzer, Frankfurt/M. J.H. Holzner, Wien

H. Huber, Innsbruck HJ. Illiger, Oldenburg N. Jaeger, Bonn W.F. Jungi, St. Gallen M.

Kaufmann, Heidelberg U.R. Kleeberg, Hamburg B. Kornhuber, Frankfurt/M. H. Löffler, Kiel H.

Ludwig, Wien U. Mohr, Hannover K. Munk, Heidelberg G.A. Nagel, Vitikon-Zürich A.

Pfleiderer, Freiburg F. Porzsolt, Ulm K. Possinger, München W. Queißer, Mannheim

P. Schlag, Heidelberg

H.-J. Schmoll, Hannover

M. Schroder, Kassel

S. Seeber, Essen

W. Vahlensieck, Bonn

M. Wannenmacher, Heidelberg

HJ. Weh, Hamburg

W. Wilmanns, München

W. Wrba, Wien 


\section{Anzeigen}

S. Karger Verlag für Medizin und Naturwissenschaften GmbH, Postfach 17 24, D-8034 Germering, Telefon (0 89) 8430 35. Gültig ist die Preisliste Nr. 6 vom 1. 10.1990.

Für den Inhalt außerhalb des redaktionellen Teiles (insbesondere Anzeigen, Industrieformationen, Pressezitate und Kongreßinforma-tionen usw.) übernehmen Schriftleitung, Beirat und Verlag keine Gewähr.

Eine Markenbezeichnung kann warenzeichengeschützt sein, auch wenn bei ihrer Verwendung in dieser Zeitschrift das Zeichen ${ }^{\circledR}$ oder ein anderer Hinweis auf etwa bestehende Schutzrechte fehlen sollte. Für Satzfehler, insbesondere bei Dosierungsangaben, wird keine Gewähr übernommen.

AUe Rechte, insbesondere das Recht der Vervielfältigung und Mikrokopie sowie der Übersetzung, vorbehalten. Nachdruck, auch auszugsweise, nur mit Genehmigung des Verlages. Fotokopien dür-fen nur für den persönlichen Gebrauch als Einzelkopie hergestellt werden. Jede im Bereich eines gewerblichen Unternehmens zulässig hergestellte oder benutzte Kopie dient gewerblichen Zwecken gem. § 54(2) UrhG und verpflichtet zur Gebührenzahlung an die Verwertungsgesellschaft WORT, Abt. VG Wissenschaft, Goethestraße 49, D-8000 München 2.

(C) Copyright 1991 by S. Karger,

Verlag für Medizin und Naturwissenschaften $\mathrm{GmbH}$,

Postfach 17 24,

D-8034 Germering (BRD)

ISBN 3-8055-5498-2

Verlagsleitung: Manfred R. Just Redaktionsassistenz: Anne Schieber Anzeigenverkauf: Marlene Scholz Anzeigenverwaltung: Eva M. Lindenau Herstellung: Horst H. Bruch Vertrieb: Gabriela Taube

Satz und Druck: Walter Biering GmbH Grafischer Betrieb Freisinger Landstraße 21 D-8000 München 45 (BRD)

Ladenpreis dieses Sonderheftes DM 18,- inkl. MwSt; Abonnenten erhalten es unberechnet. Bezugspreís der Zeitschrift:

Für Jahrgang 14,1991, DM 148,-/öS 1036,-/SFr 116,-.

1 Einzelheft kostet DM 28,-/öS 196,-/SFr 22,- einschließlich MwSt.

zuzüglich Postgebühren.

KARGER 